\title{
Investigating the Fluid Seal of Supraglottic Airway Devices in Humans Using Indicator Dye via the Drainage Tube: A Potential Roadmap for Future Studies*
}

\author{
Sebastian G. Russo", Stephan Cremer, Ulrike Mühlhäuser, Christoph Eich, Michael Quintel, \\ Martin Bauer \\ Department of Anaesthesiology, Emergency and Intensive Care Medicine, University of Göttingen Medical Centre, Göttingen, Germany. \\ Email: \#s.russo@medizin.uni-goettingen.de
}

Received January $4^{\text {th }}$, 2012; revised January $25^{\text {th }}, 2012$; accepted February $4^{\text {th }}, 2012$

\begin{abstract}
Background: The fluid seal of supraglottic airway devices (SGA) protects the airway from fluid contamination. We evaluated the suitability of indicator dye placement in the upper digestive tract of anesthetized patients combined with fiberoptical tracing to investigate the fluid seal of SGA. Methods: Patients swallowed a capsule of indigo carmine green (ICG) prior to induction of anaesthesia. After induction of anesthesia, one of two different SGA (either an i-Gel ${ }^{\mathrm{TM}}$ or an LMA-Supreme ${ }^{\mathrm{TM}}$ (LMA-S)) was inserted after randomization. Methylene blue stained normal saline was injected through the proximal opening of drainage tube during mechanical ventilation as well as spontaneous breathing. We monitored regurgitation of ICG with a flexible fiberscope (FO) inserted through the drainage tube and checked for the appearance of methylene blue in the mask bowl with the FO inserted through the airway tube. Results: In thirty-six patients with an i-Gel ${ }^{\mathrm{TM}}$ and 37 with a LMA-S no regurgitation of ICG was observed at the level of the upper oesophageal sphincter (UES). Methylene blue stained saline was not visible in any patient during pressure-controlled ventilation, but was detected in two of the 36 patients with the i-Gel ${ }^{\mathrm{TM}}$ during spontaneous breathing. Conclusion: Instilling dye through the drainage tube of SGA models with a built-in drainage tube represents a useful method to examine and to compare the fluid seal of different SGA. Our protocol presented in this study proved to be an easy and reproducible approach for future studies. Furthermore, the clinical results gained during this evaluation highlight the necessity for further investigations regarding the fluid seal competencies of SGAs in humans under clinical conditions.
\end{abstract}

Keywords: Laryngeal Mask Airway; Supraglottic Airway; Aspiration

\section{Introduction}

Supraglottic airway devices (SGAs) are widely used in anaesthesiology. Modern, gastric access SGAs are designed to separate the respiratory and the gastrointestinal tract [1]. They have an integrated drainage tube that is intended to channel regurgitated gastric fluids and gases, and to allow the insertion of a gastric tube to actively or continuously evacuate gastric contents. This represents a likely important safety improvement over classic laryngeal mask airways [2].

When using SGAs, two different seals have to be considered: The first is the gas seal that determines the ventilation efficiency of the SGA and is expressed as the leak pressure (LP). The second is the fluid seal that protects the airways against regurgitated fluids. The latter is

\footnotetext{
"There are no conflicts of interest.

${ }^{\#}$ Corresponding author.
}

closely related to the seal formed by the distal end of the SGA with the hypopharynx and the upper oesophageal sphincter (UES). The fluid seal has been studied in cadavers $[3,4]$, but unfortunately, the results cannot be fully transferred to living humans, who have pharyngeal, laryngeal as well as oesophageal muscle tone and a higher body temperature.

The aim of this evaluation was to examine whether fiberoptically tracing of indicator dyes placed in the upper digestive tract may be a suitable alternative to investigate and to compare the fluid seal competencies of SGAs in anesthetized living subjects.

\section{Methods}

\subsection{Selection and Description of Patients}

With the approval of our institutional ethics committee and written informed patient consent, we recruited elec- 
tive patients for this prospective, randomized evaluation at the University Medical Hospital, Göttingen, Germany. Inclusion criteria were: age $>18$ years, American Society of Anesthesiologists (ASA) status I or II, and a scheduled elective intervention in supine position with a predicted duration of anaesthesia of 60 minutes or more. Patients were excluded if they met one of the following criteria: laparoscopic surgery, BMI > $35 \mathrm{~kg} / \mathrm{m}^{2}$, known or predicted difficult airways, known gastro-oesophageal reflux under continuous medication.

A computer-generated randomization list was created using an online program (www.randomizer.org). For concealment, individually sealed opaque envelopes containing the name of the airway device were prepared.

\subsection{Anesthesia}

In addition to their routine premedication (7.5 mg midazolam p.o.) given 30 minutes prior to induction of anaesthesia, all patients also swallowed a capsule of $50 \mathrm{mg}$ indigo carmine green (ICG) to stain their gastric contents. Anesthesia was induced according to our institutional routine with sufentanil $(0.3-0.5 \mu \mathrm{g} / \mathrm{kg})$ and propofol (1.5 - $2.5 \mathrm{mg} / \mathrm{kg}$ ) and initially maintained with a propofol infusion (5 - $8 \mathrm{mg} / \mathrm{kg} / \mathrm{h})$. In order to facilitate spontaneous ventilation during the course of the study (see below) the propofol infusion was subsequently switched to sevoflurane (1.5 - $2.5 \mathrm{vol} \%)$.

\subsection{Airway Management and Ventilation}

We used the i-Gel ${ }^{\mathrm{TM}}$ (Intersurgical Ltd.) and the LMASupreme $^{\mathrm{TM}}$ (LMA-S; The Laryngeal Mask Company Ltd.) as they represent two relatively new SGAs with an in-built drainage channel to evaluate the presented method. The $\mathrm{i}-\mathrm{Gel}^{\mathrm{TM}}$ is a non-inflatable SGA with a gel cuff [5]. The LMA-S is a disposable, pre-curved modification of the LMA-ProSeal ${ }^{\mathrm{TM}}$ (PLMA, The Laryngeal Mask Company Ltd.) [6]. Both devices are regularly used in our institution for both training and routine airway management.

A senior anaesthesia registrar (SC), skilled in placing and using both devices, performed all cases and inserted the SGA. Insertion was attempted after an adequate depth of anaesthesia had been verified by performing a forced jaw thrust manoeuvre [7], size selection and insertion techniques of the SGAs were based on the manufacturer's recommendations. After insertion, the LMA-S devices were inflated to a cuff pressure of $60 \mathrm{~cm} \mathrm{H}_{2} \mathrm{O}$.

Correct device placement was evaluated clinically as well as fiberoptically. Clinical criteria were as follows: no air leakage through the drainage tube during positive pressure ventilation [8], ability to introduce a suction catheter (12Ch for the $\mathrm{i}-\mathrm{Gel}^{\mathrm{TM}}$, 16Ch for the LMA-S) beyond the tip of the device and establishment of successful ventilation. After the insertion was rated successful based on clinical measures, fiberoptic (FO) evaluation of the SGA's position was performed and assessed using a previously used four-point score ( 1 = only vocal cords seen; 2 = cords and/or arytenoids seen; 3 = only epiglottis seen; 4 = other (e.g. cuff, pharynx, etc)) [9].

During controlled ventilation the patients' lungs were ventilated to a target et $\mathrm{CO}_{2}$ of 35 - $40 \mathrm{mmHg}$ with pressure-controlled ventilation (PCV), a positive end-expiratory pressure (PEEP) of $3 \mathrm{~cm} \mathrm{H}_{2} \mathrm{O}$, a respiratory rate between 14 and $16 \mathrm{~min}^{-1}$ and an inspiratory to expiratory ratio of $1: 1.5$ with a target tidal volume of $7 \mathrm{ml} / \mathrm{kg}$.

The SGAs were taped from left to right across the maxilla as indicated by the manufacture to keep them in their intended position.

\subsection{Fluid Seal Evaluation}

During the course of the intervention, the appearance of regurgitated ICG-stained gastric content was evaluated every 15 minutes by observing the distal end of the drainage tube and the mucosa of the upper oesophageal sphincter with a flexible fiberscope (2.5 mm OD). If no regurgitation was seen at this level after 30 minutes, a gastric tube was inserted into the stomach to inspect for and then remove all stained gastric fluids. The gastric tube was then removed.

Subsequently, methylene blue stained normal saline $(10 \mu \mathrm{g} / \mathrm{ml})$ was instilled under continuous fiberoptic observation of the mask bowl into the drainage tube of the SGA. In order to avoid an airlock in the drainage tube and to assure that the drainage tube was entirely filled, saline was instilled through an olive-tipped cannula inserted into the drainage tube. Saline was instilled until the drainage tube was entirely filled and seen at the proximal end of the drainage tube (Figure 1).

In vitro testing revealed the minimum volume needed to fill the entire drainage tube (e.g. size four devices $5 \mathrm{ml}$ for the $\mathrm{i}-\mathrm{Gel}^{\mathrm{TM}}$ and $7.5 \mathrm{ml}$ for the LMA-S). If more saline was needed-to fill the hypopharynx or because saline was drained via the oesophagus_-additional saline was instilled until the column of saline stressed the fluid seal for 30 seconds. This procedure was performed twice, first during mechanical ventilation and then during spontaneous breathing.

At the end of anaesthesia a gastric tube (12Ch for the i-Gel ${ }^{\mathrm{TM}}$, 16Ch for the LMA-S) was again inserted into the stomach to remove all gastric fluids prior to removing the SGA.

\subsection{First Seal Measurement}

The leak pressure (LP) was measured as previously de- 


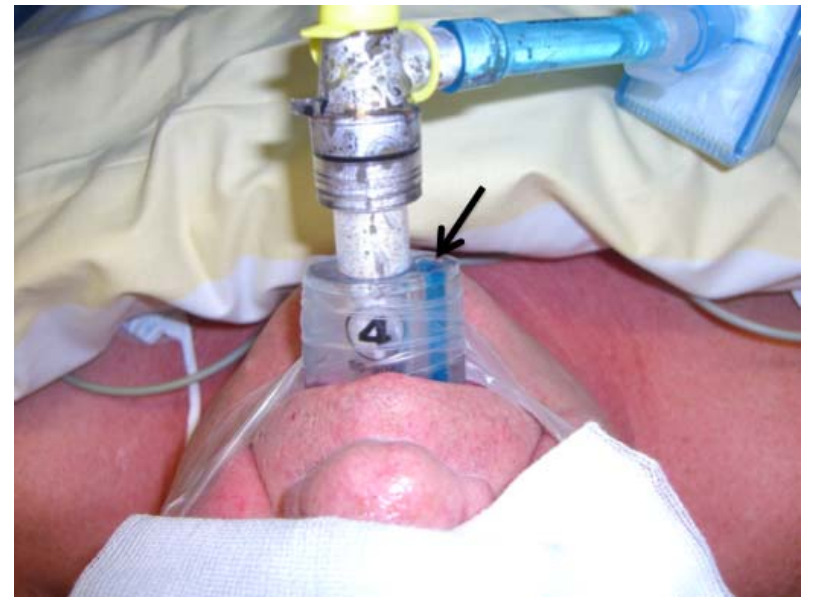

Figure 1. I-Gel ${ }^{\mathrm{TM}}$ (Intersurgical Ltd., Wokingham, UK) in position. The black arrow indicates the distal end of the fluid column of methylene blue stained saline in the drainage tube.

scribed [10]. Briefly, an air-leak was sought using a stethoscope placed over the larynx with airway pressure gradually increased with a continuous oxygen flow low of $3 \mathrm{l} / \mathrm{min}$ with the pop-off valve of the anaesthesia circuit set to a maximum of $35 \mathrm{~cm} \mathrm{H}_{2} \mathrm{O}$. The airway pressure at which an audible leak was auscultated was defined as the LP.

\subsection{Statistical Analysis}

All data were documented in an Excel ${ }^{\mathrm{TM}}$ spreadsheet (Microsoft Excel 2008) and analyzed using SPSS Statistics $^{\text {TM }}$ (IBM SPSS Inc., Chicago, IL, USA). A univariate t-test for independent groups was used to compare the biometrical data as well as the duration of anaesthesia. Chi-square test was used to compare the incidence of methylene blue occurrence in the mask bowl. Data are presented as mean and standard deviation.

Due to the exploratory nature of this evaluation no sample size calculation was performed prior to start the evaluation.

\section{Results}

Forty patients were recruited for each device. The biometric data (BMI $26.0 \pm 4.2 \mathrm{~kg} / \mathrm{m}^{2}, p=0.57$; height 172 $\pm 10 \mathrm{~cm}, p=0.97$; age $48 \pm 16$ years, $p=0.53$; ratio female to male: i-Gel ${ }^{\mathrm{TM}} 24: 16$, LMA-S 26:14, $p=0.47$ ) as well as the duration of anaesthesia (119 \pm 47 minutes, $p$ $=0.75$ ) did not differ between groups. Two patients in the $\mathrm{i}-\mathrm{Gel}^{\mathrm{TM}}$ group and one patient in the LMA-S group had to be removed from the analysis because the wrong size of the device had been used. Based on the criteria defined, SGA insertion was unsuccessful in two of 38 patients with the $\mathrm{i}-\mathrm{Gel}^{\mathrm{TM}}$ (5.5\%) and in two of 39 with the LMA-S (5\%). Therefore, 36 patients in the $\mathrm{i}-\mathrm{Gel}^{\mathrm{TM}}$ and 37 patients in the LMA-S group proceeded with the evaluation.

\subsection{First Seal Evaluation}

The LP did not differ between the two devices: i-Gel ${ }^{\mathrm{TM}}$ $25.9 \pm 5.6 \mathrm{~cm} \mathrm{H}_{2} \mathrm{O}$ vs. LMA-S $26.1 \pm 5.1 \mathrm{~cm} \mathrm{H}_{2} \mathrm{O}(p=$ 0.8 ). Gastric inflation was not noted.

\subsection{Fiberoptic Assessment}

The fiberoptic view was grade 2 (cords and arytenoids visible) for all patients. Furthermore, the tip of both devices was always located distal to the arytenoids, inserting into the hypopharynx (Figures 2(a) and (b)).

\subsection{Fluid Seal Evaluation}

The gastric content of all patients was stained with ICG. No signs of ICG regurgitation were seen.

Stained saline was not detected around the arytenoids or in the mask bowl in any of the LMA-S patients, but it was observed in two of the 36 patients with an $\mathrm{i}-\mathrm{Gel}^{\mathrm{TM}}$ device during spontaneous breathing $(5.5 \%, p=0.148$; Figure 3). The dye was easily suctioned from the mask bowl as soon as it appeared and none was seen to pass into the glottis.

\section{Discussion}

Despite data from cadaver studies [3,4], little is known about the effectiveness of the fluid seal of SGAs in living human subjects. A protocol to investigate the ability to protect the airways from contamination therefore seems warranted [11].

Evens et al. instilled stained saline into the drainage tube of the PLMA in order to investigate the ability of the device to protect the airway against fluids [12]. We combined the dye instillation through the drainage tube with a modification of the method previously described by Lallo et al. who stained the gastric contents with methylene blue [13]. We stained the gastric contents with ICG in order to be able to visually differentiate green material coming from the lower esophagus and methylene blue stained saline instilled into the drainage tube. In contrast to Lallo et al. we found no regurgitation of stained gastric content. The results gained by Lallo and colleagues are somewhat surprising, as regurgitation occurred in up to $14 \%$ of their patients and methylene blue was also found at the proximal end of the mask bowl.

In vitro experiments conducted prior to the present trial with acidified methylene blue and ICG at $36^{\circ} \mathrm{C}$, showed that indicator dyes do not tend to adhere to the surface of the PLMA, LMA-S or i-Gel ${ }^{\mathrm{TM}}$. Furthermore, 

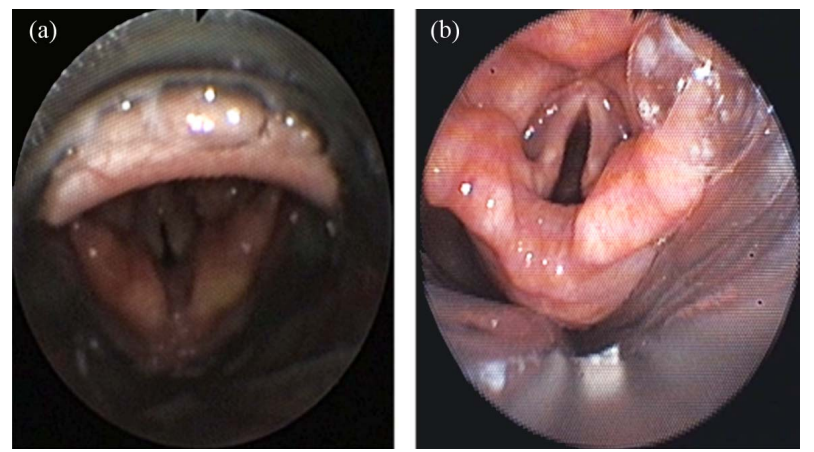

Figure 2. Fiberoptic view of the supraglottic airway devices in situ. (a) $\mathrm{i}-\mathrm{Gel}^{\mathrm{TM}}$; (b) LMA-Supreme ${ }^{\mathrm{TM}}$.

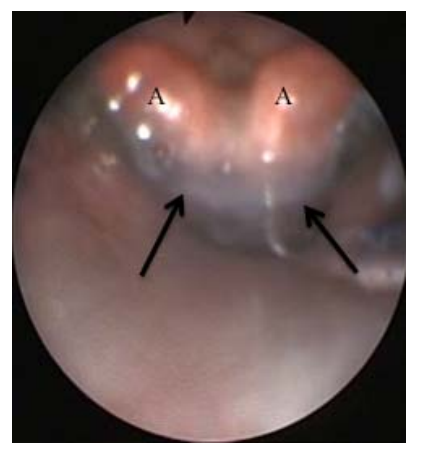

Figure 3. Example of stained saline entering the mask bowl. Arrow: Dye around the arytenoids (A).

regurgitation in elective patients is considered a fairly rare occurrence. Indeed, although we acknowledge the small number of patients, no regurgitation occurred during the presented study. Therefore, we believe that an in vivo fluid seal stress test, such as the installation of stained saline into the drainage tube seems to be a more reliable method to investigate the fluid seal of SGAs in humans than staining the gastric content or performing cadaver studies.

Interestingly, the approach to instil dye into the drainage tube, to the best of our knowledge first and solely described by Evens et al. [12] has not been used in other investigations. In contrast to Evans at al. our protocol contained several modifications. First, we investigated the fluid seal during controlled and spontaneous ventilation in one and the same patients and not in two independent groups. This allows one to investigate potential differences of the fluid seal comparing controlled and spontaneous ventilation. Interestingly, the cases of fluid seal leakage in the study by Evans et al. as well as in the presented study occurred during the phases of spontaneous ventilation. Secondly, we instilled stained saline at two distinct periods during controlled and spontaneous ventilation and not during the entire time of SGA aided ventilation. Thirdly, during dye installation the airways were continuously observed fiberoptically. As leakage of the fluid seal has been observed in 2\% (Evans et al. [12]) and 5\% (this study) the latter protocol may represent a potentially safer approach in order to avoid accidental aspiration of dye.

Based on FO evaluation, the anatomical position of the $\mathrm{i}-\mathrm{Gel}^{\mathrm{TM}}$ and the LMA-S were identical (see also Figure 2). However, the accuracy of the FO evaluation is limited by the difficulties in precisely localizing the distal tip of the SGA device. The view from above with the fiberscope did not allow one to confidently identify the anatomical structures where the distal end of the SGA was located: hypopharynx, UES or esophagus. Thus, the fluid seal competence cannot be evaluated by fiberoptic evaluation.

This study was not intended to detect small differences between the two devices, but to assess the dye indicator method for testing fluid seal competencies in humans. Due to the small number of patients in each group and the low incidence of the observed dye leak, one cannot confidently draw any conclusions with regard to a possibly increased risk of aspiration during spontaneous ventilation with the $\mathrm{i}-\mathrm{Gel}^{\mathrm{TM}}$. The observed leakage incidence of two out of 36 for the $\mathrm{i}-\mathrm{Gel}^{\mathrm{TM}}$ is well within the calculated 95\% confidence interval (CI) for a leak with the LMA-S. In order to draw robust conclusions, a larger study is required to test for a statistically significant difference between the two devices with regard to their fluid seal competencies. Considering the $95 \%$ CI of $0 \%-9 \%$ for the incidence of a fluid seal leakage of the LMA-S calculated from our data, a minimum of 200 patients per group would be required to prove that the observed difference between the two devices was statistically significant. Nonetheless, regarding the LP, our study confirms previous results regarding the first seal qualities of the LMA-S and the i-Gel ${ }^{\mathrm{TM}}[5,14]$.

In conclusion, installation of stained saline into the drainage tube allows to investigate and to compare the fluid seal of SGAs with a built-in drainage tube. The protocol presented in this study may serve as possible roadmap for further investigations as it represents a simple, reproducible and safe routine. In contrast, staining the gastric content does not seem to be a helpful approach for the intended purpose in elective patients.

\section{Authorship Attribution}

SGR designed the study, performed the experiments, conducted the statistical analyses, interpreted the data, and drafted the manuscript. SC inserted the SGA, helped to interpret the data and to draft the manuscript. UM helped during study planning and manufactured the indigo carmine green capsules. CE, MQ and MB provided intellectual as well logistical support during the study and drafted the manuscript. 


\section{REFERENCES}

[1] T. M.Cook and B. Howes, "Supraglottic Airway Devices: Recent Advances," Continuing Education in Anaesthesia, Critical Care \& Pain, Vol. 11, No. 1, 2011, pp. 1-33.

[2] T. M. Cook, G. Lee and J. P. Nolan, "The ProSeal ${ }^{\mathrm{TM}}$ Laryngeal Mask Airway: A Review of the Literature," Canadian Journal of Anaesthesia, Vol. 52, No. 7, 2005, pp. 739-760. doi:10.1007/BF03016565

[3] W. Schmidbauer, S. Bercker, T. Volk, G. Bogusch, G. Mager and T. Kerner, "Oesophageal Seal of the Novel Supralaryngeal Airway Device i-Gel in Comparison with the Laryngeal Mask Airways Classic and ProSeal ${ }^{\mathrm{TM}}$ Using a Cadaver Model," British Journal of Anaesthesia, Vol. 102, No. 1, 2009, pp. 135-139. doi:10.1093/bja/aen319

[4] S. Bercker, W. Schmidbauer, T. Volk, G. Bogusch, H. P. Bubser, M. Hensel and T. Kerner, "A Comparison of Seal in Seven Supraglottic Airway Devices Using a Cadaver Model of Elevated Esophageal Pressure,” Anesthesia \& Analgesia, Vol. 106, No. 2, 2008, pp. 445-448. doi:10.1213/ane.0b013e3181602ae1

[5] J. J. Gatward, T. M. Cook, C. Seller, J. Handel, T. Simpson, V. Vanek and F. Kelly, "Evaluation of the Size 4 i-Gel ${ }^{\mathrm{TM}}$ Airway in One Hundred Non-Paralysed Patients," Anaesthesia, Vol. 63, No. 10, 2008, pp. 1124-1130. doi:10.1111/j.1365-2044.2008.05561.x

[6] A. van Zundert and J. Brimacombe, "The LMA Supreme-A Pilot Study,” Anaesthesia, Vol. 63, No. 2, 2008, pp. 209-210. doi:10.1111/j.1365-2044.2007.05421.x

[7] R. Townsend, J. Brimacombe, C. Keller, V. Wenzel and H. Herff, "Jaw Thrust as a Predictor of Insertion Conditions for the Proseal Laryngeal Mask Airway,” Middle East Journal of Anesthesiology, Vol. 20, No. 1, 2009, pp.
59-62.

[8] J. Brimacombe and C. Keller, "Prime the ProSeal ${ }^{\mathrm{TM}}$ Drain Tube with Lube from a Tube!” Canadian Journal of Anaesthesia, Vol. 52, No. 3, 2005, pp. 338-339. doi:10.1007/BF03016079

[9] C. Verghese, J. Berlet, A. Kapila and R. Pollard, "Clinical Assessment of the Single Use Laryngeal Mask Airway- the LMA-Unique,” British Journal of Anaesthesia, Vol. 80, No. 5, 1998, pp. 677-679.

[10] C. Keller, J. R. Brimacombe, K. Keller and R. Morris, "Comparison of Four Methods for Assessing Airway Sealing Pressure with the Laryngeal Mask Airway in Adult Patients," British Journal of Anaesthesia, Vol. 82, No. 2, 1999, pp. 286-287. doi:10.1093/bja/82.2.286

[11] B. Gibbison, T. M. Cook and C. Seller, "Case Series: Protection from Aspiration and Failure of Protection from Aspiration with the i-Gel Airway," British Journal of Anaesthesia, Vol. 100, No. 3, 2008, pp. 415-417. doi:10.1093/bja/aem396

[12] N. R. Evans, S. V. Gardner and M. F. James, "ProSeal Laryngeal Mask Protects against Aspiration of Fluid in the Pharynx,” British Journal of Anaesthesia, Vol. 88, No. 4, 2002, pp. 584-587. doi:10.1093/bja/88.4.584

[13] A. Lallo, P. Drolet and M. Lacroix, "Performance of the PAXpress $^{\mathrm{TM}}$ vs the ProSeal ${ }^{\mathrm{TM}}$ Laryngeal Mask Airway during General Anesthesia," Canadian Journal of Anaesthesia, Vol. 54, No. 1, 2007, pp. 28-33. doi:10.1007/BF03021896

[14] A. Timmermann, S. Cremer, C. Eich, S. Kazmaier, A. Brauer, B. M. Graf and S. G. Russo, "Prospective Clinical and Fiberoptic Evaluation of the Supreme Laryngeal Mask Airway,” Anesthesiology, Vol. 110, No. 2, 2009, pp. 262-265. 